\title{
ПРОЦЕССЫ РАЗЛОЖЕНИЯ ХЛОРПРОИЗВОДНЫХ ФЕНОЛА ПОД ДЕЙСТВИЕМ ЭЛЕКТРИЧЕСКИХ РАЗРЯДОВ
}

\author{
А. С. Манукян, В. В. Рыбкин \\ АННА СЛАВИКОВНА МАНУКЯН - к.т.н., заведующзая объединенной редакцией научных журналов Ива- \\ новского государственного химико-технологического университета.E-mail: annamanuk77@gmail.com.
}

\begin{abstract}
ВЛАДИМИР ВЛАДИМИРОВИЧ РЫБКИН - д.х.н., профессор, главный научный сотрудник кафедры технологии приборов и материалов электронной техники Ивановского государственного химико-технологического университета.E-mail: rybkin@isuct.ru.
\end{abstract}

153000, пр. Шереметевский, д. 7, Иваново. Ивановский государственный химико-технологический универcumem.

\begin{abstract}
Проведено аналитическое рассмотрение процессов, протекающих при действии различньх типов электрических разрядов атмосферного давления на водные растворы хлорпроизводных фенола. Показано, что из всех исследованных разрядов диэлектрический барьерный разряд в кислороде является наиболее эффективным. Он обеспечивает 100 \% деструкцию при высоких скоростях разложения и хорошие энергетические показатели. Другие методы AOP(Advance Oxidative Processes, УФ-воздействие, реагенты Фентона, реагенты Фентона с УФ излучением) существенно уступают методам, с использованием разрядов по скоростям разложения и энергетическим эффективностям. Рассмотрено влияние различных параметров разряда, катализаторов, вида плазмообразующего газа на эффективность деструкции. Приведень данные о продуктах деструкиии хлорфенолов, на основании которых предложены механизмы процессов деградации, протекающих под действием разряда. В общих чертах механизм подобен механизму разложения фенола.
\end{abstract}

Ключевые слова: электрический заряд, производные хлорфенола, разложение.

\section{PROCESSES OF DECOMPOSITION OF CHLORINE DERIVATIVES OF PHENOL UNDER THE ACTION OF ELECTRIC DISCHARGES}

\author{
A.S. Manukyan, V.V. Rybkin \\ 7, Sheremetievskiy Av., Ivanovo, 153000, Russia. Ivanovo state university of chemistry and technology.
}

An analytical review of the processes occurring under the action of various types of electric discharges of atmospheric pressure on aqueous solutions of chlorine derivatives of phenol was carried out. It is shown that of all the discharges studied, the dielectric barrier discharge in oxygen is the most effective. It provides $100 \%$ destruction at high decomposition rates and good energy parameters. Other AOP methods (Advance Oxidative Processes, UV exposure, Fenton reagents, Fenton reagents with UV radiation) are significantly inferior to methods using discharges in terms of decomposition rates and energy efficiency. The influence of various parameters of the discharge, catalysts, and the type of plasma-forming gas on the efficiency of destruction is considered. Data on the products of the destruction of chlorophenols are presented, on the basis of which the mechanisms 
of degradation processes occurring under the action of a discharge are proposed. In general terms, the mechanism is similar to phenol decomposition mechanism.

Keywords: electric discharge, phenol chlorine derivatives, decomposition.

Хлорфенолы представляют собой особую группу приоритетных токсичных загрязнителей, перечисленных ЕРА США в Законе о чистой воде и Европейским решением 2455/2001/ЕС, так как большинство из них токсичны и слабо поддаются биологическому разложению. Их трудно удалить из окружающей среды период полураспада в воде может достигать 3,5 месяцев в аэробных водах и нескольких лет в органических отложениях. Из-за многочисленных использований их находят в грунтовых водах, сточных водах и почве и даже в трофических цепях мест с очень низким уровнем загрязнения [1].

Из-за высокой стабильности фенольных производных хлора плазменная обработка воды является очень перспективным инструментом для их деградации.

В данной работе будут рассмотрены немногочисленные результаты по данному вопросу, касающие кинетики разложения хлорпроизводных бензола, образующихся продуктов и возможных механизмах реакций.

Обработка раствора пентахлорфенола диэлектрическим барьерным разрядом (ДБР) атмосферного давления в кислороде обеспечивает эффективность разложения $65 \%$ [2]. Тот же результат был достигнут в кислородном ДБР в работе [3].

Почти 100 \% степень разложения 2,4дихлорфенола была получена в кислородном ДБР падающего потока в исследовании [4].

Использование разряда скользящей дуги в кислороде с распылением раствора дало эффективность разложения 4хлорфенола 82 \% при времени обработки 80 мин [5].

Стримерный подводный разряд в пузырьках газа в сочетании с катализатором $\mathrm{TiO}_{2}$ (порошок, анатаз) показал эффективность $100 \%$ при обработке раствора 4хлорфенола при времени обработки 30 мин [6].

Деградации водных растворов для шести изомеров трихлорфенола и шести изомеров дихлорфенола была исследована в работе [7] для анодного контактного электролиза с тлеющим разрядом (ACGDE). Кинетика была даже медленнее, чем в случае разряда стримерных пузырьков, упомянутого выше. Эффективность разложения $100 \%$ была достигнута при времени обработки 150 мин.

Кинетические закономерности разложения, которые позволяют также оценить энергетиче- скую эффективность, изучены крайне слабо. В тех работах, где это было исследовано [4, 7] было обнаружено, что разложение протекает по 1-му кинетическому порядку по концентрации соответствующего хлорфенола. Так в работе [7] при действии ACGDE мощностью 35 Вт; объеме раствора 70 мл и начальной концентрация - 5 ммоль/л кинетика была довольно медленной. Константа скорости составляла $4,3 \cdot 10^{-4} \mathrm{c}^{-1}$, так что степень разложения $90 \%$ была достигнута за 55 мин. А энергетическая эффективность составила $1,53 \cdot 10^{-2}$ разложившихся молекул на 100 эВ вложенной энергии. В работе [4] (ДБР в кислороде) показатели были существенно выше. Константа скорости составляла $2 \mathrm{c}^{-1}$, энергетическая эффективность 0,17 на 100 эВ вложенной энергии, так что степень разложения достигала $100 \%$. Степени разложения увеличивались с ростом мощности, вложенной в разряд, как и скорости разложения.

Интересно сравнить эти показатели с данными для фенола и хлорбензола. Типичные значения энергетической эффективности фенола находятся в диапазоне (1-10)·10-3 мол/100 эВ [8, 9]. Для хлорбензола максимальное значение $0,08\left(\mathrm{~K}=5 \cdot 10^{-3} \mathrm{c}^{-1}\right)$ было получено в исследовании [10], где применялся ACGDE. Кажется, что фенол более стабилен, чем хлорбензол, и последний, в свою очередь, более стабилен, чем 2,4дихлорфенол, для действия разряда.

Кроме того, мы можем сравнить результаты плазменной деградации с результатами, полученными другими методами AOP (Advance Oxidative Processes). Так, обработка растворов УФфотолизом дает константы скорости, изменяющиеся в диапазоне (2,9-173) $10^{-3} \mathrm{c}^{-1}$ при начальных концентрациях 2,4дихлорфенола 0,3-0,5 ммоль/л в диапазоне длин волн 185-436 нм [11, 12]. Использование реагента Фентона $\left(\mathrm{H}_{2} \mathrm{O}_{2}\right.$ и соли железа (II)) дает константы скорости (3,5-17) $10^{-3} \mathrm{c}^{-1}$ при начальных концентрациях 2,4дихлорфенола $0,3-0,5$ ммоль/л $[13,14]$. При той же концентрации при действии озона наблюдается самая низкая константа скорости $4 \cdot 10^{-4} \mathrm{c}^{-1}$, в то время как комбинированное действие $\mathrm{O}_{3}$ и УФ происходит с константой скорости $1,1 \cdot 10^{-3} \mathrm{c}^{-1}$ [14]. Сравнение упомянутых выше данных с константами скорости, полученными в работе [4], показывает, что ДБР в кислороде имеет очевидные преимущества по сравнению с другими методами АОР. 
Константы скорости разложения 4-хлорфенола в различных газах. Импульсный стримерный разряд в пузырьках газа. Напряжение 14 кВ. Данные работы [7]

\begin{tabular}{lcccccc}
\hline \multicolumn{1}{c}{ Газ } & $\mathbf{N}_{2}$ & $\mathbf{N}_{2}$ & $\mathbf{O}_{2}$ & $\mathbf{O}_{2}$ & Воздух & Воздух \\
\hline $\mathrm{TiO}_{2}$, мГ/л & 0 & 50 & 0 & 50 & 0 & 50 \\
Константа скорости, $10^{-3} \mathrm{c}^{-1}$ & 0,57 & 0,79 & 1,56 & 2,81 & 0,72 & 1,07 \\
\hline
\end{tabular}

$\begin{array}{ccccc}\text { Хлорфенол } \rightarrow \text { Гидроксипроизводные } \rightarrow \quad \text { Карбоновые кислоты } & \rightarrow \quad \text { Альдегиды } \\ & & & \downarrow \\ & & \text { Двуокись углерода } & \leftarrow & \downarrow\end{array}$

Рис. Возможная схема деградации хлорфенолов

В случае надводных разрядов, горящих в контакте с раствором, эффективность разложения зависит от сорта плазмообразующего газа. Разряд в кислороде оказывается более эффективным, чем в азоте или воздухе (Таблица).

Применение катализаторов как гетерогенных $\left(\mathrm{TiO}_{2}[7]\right)$, так и гомогенных (соли $\left.\mathrm{Fe}^{2+},[5,15]\right)$ так же ускоряют процесс разложения. Но их использование усложняет технологию очистки, так как необходимо вводить дополнительные операции удаления катализаторов.

Знание продуктов разложения является важным, так как они определяет токсичность обработанного раствора. Эти же сведения необходимы для выяснения механизма химических превращений. Такие данные получены в работах [4, 7]. При разложении 2,4дихлорфенола на начальном этапе наблюдалось образование 1,4гидрохинона и 1,4бензохинона в результате замещения хлора группой ОН [7]. Замена ОНгруппы в орто-положении по отношению к фенольной ОНгруппы происходила одновременно с образованием хлоркатехинов. Далее, эти соединения превращались в карбоновые кислоты, альдегиды и диоксид углерода. В растворе также образовывались хлорид-ионы $\mathrm{Cl}^{-}$, а молекулы $\mathrm{Cl}_{2}$ появлялись в газовой фазе разряда [4]. Полученные закономерности разрушения аналогичны наблюдаемым при деградации фенола и его гидроксипроизводных $[16,17]$. Образуются те же гидроксипроизводные фенола, карбоновые кислоты, альдегиды и диоксида углерода. В [16] было показано, что разложение гидроксипроизводных приводит к образованию карбоновых кислот, альдегидов и $\mathrm{CO}_{2}$. В свою очередь, разложение карбоновых кислот [18] дает альдегиды. Разложение альдегидов приводит к образованию только углекислого газа $[19,20]$. Поэтому общую схему процес- сов, протекающих в растворе, можно представить в виде, представленном на рисунке.

Судя по продуктам деградации, частицей, инициирующей первичную реакцию, являются радикалы ОН. По видимому, лимитирующей стадией процесса в целом является стадия разложения карбоновых кислот. Они имеют минимальную константу скорости взаимодействия с ОН $\left(9,2 \cdot 10^{6}\right.$ моль/(л·с) для $\mathrm{CH}_{3} \mathrm{COOH}[21], 1,4 \cdot 10^{10}$ моль/(л·с)) для фенолов [22]).

Таким образом, применение газовых разрядов является эффективным инструментом для очистки водных растворов от хлорпроизводных фенола.

\section{Литература}

1. Pera-Titus M., Garci'a-Molina V., Baños M.A, Gime'nez J, Esplugas S. Appl. Catal. B Environ. 2004. V. 47. No 4. P. 219-256.

2. Lu N., Li J., Wang X., Wang T., Wu Y. Plasma Chem. Plasma Process. 2012. V. 32. No 1. P. 109-121.

3. $Q u$ G.Z., Lu N., Li J., Wu Y., Guo-Feng Li G.F., Li D. J. Hazard. Mater. 2009. V. 172. No 1. P. 472-478.

4. Gushchin A.A., Grinevich V.I., Shulyk V.Ya., Kvitkova T.Yu., Rybkin V.V. Plasma Chem. Plasma Process. 2018. V. 38. No 1. P. 123-134.

5. Du C.M., Yan J.H., Cheron B.G. Plasma Chem. Plasma Process. 2007. V. 27. No 2. P. 635-646.

6. Hao X.L., Zhou M.H., Zhang Y., Lei L.C. Plasma Chem. Plasma Process. 2006. V. 26. No 5. P. 455-468.

7. Yang H., Caixia, Tezuka M. Plasma Chem. Plasma Process. 2013. V. 33. No 6. P. 1043-1052.

8. Манукян А.С., Рыбкин В.В. Промышленное производство и использование эластомеров. 2019. №4. C. 6-11.

9. Манукян А.С., Рыбкин В.В. Нефтегазохимия. 2019. №3-4. C. 64-67.

10. Liu Y., Jiang X. Plasma Chem. Plasma Process. 2008. V. 28. No 1. P. 15-24. 
11. Kuo W.S. Chemosphere. 1999. V. 39. No 11. P. 18531860.

12. Benı'tez F.J., Beltra'n-Heredia J., Acero J.L., Rubio F.J. J. Chem. Technol. Biotechnol. 2001. V. 76. No3. P. 312-320.

13. Tang W.Z., Huang C.P. Chemosphere. 1996. V. 33. No 8. P. 1621-1635.

14. Beni'tez F.J., Beltra'n-Heredia J., Acero J.L., Rubio F.J. Chemosphere. 2000. V. 41. No 8. P. 1271-1277.

15. Wang L. Plasma Chem. Plasma Process. 2009. V. 29. No3. P. 241-250.

16. Bubnov A.G., Burova E.Yu., Grinevich V.I., Rybkin V.V., Kim J.-K., Choi H.-S. Plasma Chem. Plasma Process. 2007. V. 27. No2. P. 177-187.
17. Bobkova E.S., Krasnov D.S., Sungurova A.V., Rybkin V.V., Choi H.-S. Korean J. Chem. Eng. 2016. V. 33. No 5. P. 1620-1628.

18. Bobkova E.S., Isakina A.A., Grinevich V.I., Rybkin V.V. Russ J. Appl. Chem. 2012. V. 85. No 1. P. 71-75.

19. Bobkova E.S., Grinevich V.I., Kvitkova E.Yu., Rybkin V.V. Izv. Vyssh. Uchebn. Zaved. Khim. Khim. Tekhnol. 2011. V. 54. No 8. P. 55-58.

20. Шутов Д.А., Иванов А.Н., Рыбкин В.В., Манукян A.C. Изв. вузов. Химия и хим. технология. 2020. Т. 63. Вып. 2. С. 91-98.

21. Thomas J.K. Trans. Faraday Soc. 1965. V. 61. P. 702-707.

22. Shutov D.A., Sungurova A.V., Choukourov A., Rybkin V.V. Plasma Chem. Plasma Process. 2016. V. 36. No 5. P. 1253-1269. 